\title{
Near Field and Optical Diffraction of 3D Dielectric Corner by Transmission Line Modeling and Multipole Expansion of Green's Function
}

\author{
Alessandro Massaro*, Roberto Cingolani, Adriana Passaseo ${ }^{\dagger}$, and Massimo De Vittorio \\ National Nanotechnology Laboratory of CNR-INFM, Distretto Tecnologico-ISUFI, Università del Salento, \\ Via Arnesano, 73100 Lecce, Italy
}

\begin{abstract}
We propose a novel modeling of the dielectric cubic corner, that is suitable for inclusion in a standard electromagnetic (EM) simulator. The model starts from a consideration of the equivalent current densities on the cube facets. It proceeds by employing a classical multipole expansion of the Green's function at the corner and introduces the novel principle of Simultaneous Transverse Resonance Diffraction (STRD) in order to determine the singularity of the EM field. The novel STRD approach considers the analogy between the EM field in proximity of the 90 deg. dielectric corner and resonant transmission lines. We combined the analytical and the numerical approaches in order to obtain an efficient numerical procedure. The theory is validated by standard finite element method (FEM) simulation, yielding information about the accuracy of the near field around the dielectric corner.
\end{abstract}

Keywords: Dielectric Corner, Optical Diffraction, Integrated Optic, Near Field.

\section{INTRODUCTION}

Recent technological developments in integrated optics have made possible, even common, the realization of quasi-planar structures with 3D dielectrics corners for nanoscale applications. These involve integrable sharp singularities of the electromagnetic (EM) field, localized around the corners, ${ }^{1-6}$ which, in turn, cause considerable difficulties for the numerical electromagnetic simulators as they force to have recourse to finer or variable meshing. ${ }^{1,2}$ Full-wave numerical techniques in time/frequency-domain like the Finite Difference in Time/Frequency Domain (FD-TD/FD) are efficient and flexible techniques for the analysis of a large class of electromagnetic problems. One of the main limitations of these and other numerical techniques is that the space-time discretization scheme fails to accurately describe the singularities of the electromagnetic field and the complex optical diffraction problems, ${ }^{3,4}$ which occur near sharp dielectric edges. Unless a very fine mesh is used, the singular behaviour around the corner is poorly represented and the frequency domain characteristics of the structure will typically be shifted. For these reasons, it is very advantageous to treat analytically with accuracy the EM field configuration in proximity of a 3D

\footnotetext{
*Author to whom correspondence should be addressed.

${ }^{\dagger}$ Permanent address: IMM-CNR sezione Lecce, University Campus, Lecce-Monteroni 73100, Italy.
}

dielectric corner (see Fig. 1(a)). In order to obtain a workable analytical model of this configuration, we employ a classical multipole expansion of the field near the corner, where the field can be considered as quasi-static ${ }^{7}$ and can be expressed by the Green's function. ${ }^{8-10}$ Moreover, for the first time, we employ the novel principle of Simultaneous Transverse Resonance Diffraction (STRD) in each of the three orthogonal coordinates around the corner. The latter principle determines univocally the order of the singularity to be used in the multipole expansion. The STRD model provides an equivalent circuit shown in Figure 1(b), suitable for inclusion in a standard full-wave electromagnetic solver in order to accurately describe structures with sharp dielectric singularities. By combining the analytical approach which gives the possibility to understand the physical aspects, and the numerical one which can provide more geometric variants of the scattered structure, we obtain an efficient numerical procedure that decrease the computational cost with a good solution accuracy. In the present contribution the new theory is validated by the use of a commercial simulators based on Finite Element Method (FEM) approach.

\section{STRD TRANSMISSION LINE MODEL}

In order to combine the diffraction of the 3D dielectric corner with the transmission line equivalent circuit of 


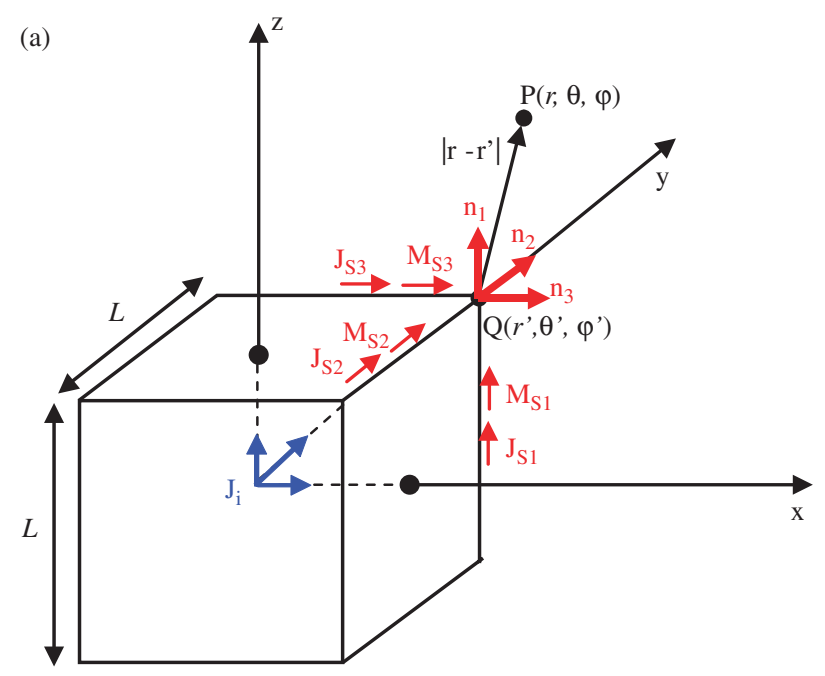

(b)

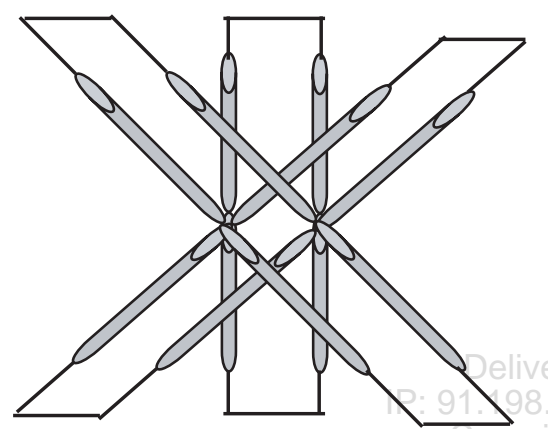

Fig. 1. (a) Equivalent electric and magnetic current densities, and (b) transmission line equivalent circuits of a $3 \mathrm{D}$ cubic dielectric corner.

Figure 1(b) we consider a transverse electric $\mathrm{TE}\left(H_{z}, E_{\rho}\right.$, $\left.E_{\phi}\right)$ polarized field defined in cylindrical coordinates as

$$
E_{\rho}=-j \omega \mu \frac{1}{\rho} \frac{\partial \psi}{\partial \phi} \quad E_{\phi}=j \omega \mu \frac{\partial \psi}{\partial \rho} \quad H_{z}=k^{2} \psi
$$

where $\psi$ is a suitable Hertzian magnetic potentials which satisfies Helmholtz equation in cylindrical coordinates. The field reported in Eq. (1) is TE with respect to the $z$-direction, briefly $\mathrm{TE}^{z}$, but is also transverse magnetic $\mathrm{TM}$ with respect to the $\phi$-direction (thus $\mathrm{TM}^{\phi}$ ). When a several dielectrics are present the field must be described by two potentials ${ }^{11}$ unless the potentials are taken in the direction normal to the stratification. In this case the stratification is normal to the $\phi$-direction. Hence, we describe the field in our structure as just $\mathrm{TM}^{\phi}$ or $\mathrm{TE}^{\phi}$. Since for a two-dimensional case $\mathrm{TE}^{z}$ coincides with $\mathrm{TM}^{\phi}$, we may refer to this field by either of these names. Near the edge we assume a potential solution of the form ${ }^{11}$

$$
\psi(\rho, \phi)=\frac{1}{k_{i}^{2}} \rho^{\nu}\left[A_{i} \sin (\nu \phi)-B_{i} \cos (\nu \phi)\right]
$$

which gives the following expression for the fields

$$
\begin{gathered}
E_{\rho}=-\frac{j \nu}{\omega \varepsilon_{i}} \rho^{\nu-1}\left[A_{i} \cos (\nu \phi)+B_{i} \sin (\nu \phi)\right] \\
H_{z}=\rho^{\nu}\left[A_{i} \cos (\nu \phi)-B_{i} \sin (\nu \phi)\right]
\end{gathered}
$$

We note that the Eq. (3) represents a pair of fields transverse to the $\phi$-direction; their ratio provides the $\mathrm{TM}^{\phi}$ characteristic impedance which may describe the $\phi$-dependence by means of an equivalent transmission line. In particular the following equivalent voltages and currents

$$
\begin{gathered}
V_{i}=\frac{1}{\varepsilon_{i}}\left[A_{i} \cos \left(\nu \phi_{i}\right)+B_{i} \sin \left(\nu \phi_{i}\right)\right] \\
I_{i}=\left[A_{i} \sin \left(\nu \phi_{i}\right)-B_{i} \cos \left(\nu \phi_{i}\right)\right] \quad i=1,2
\end{gathered}
$$

gives the EM field components, where $\nu \phi_{i}$ represent the electrical length related to the transmission line traveled by voltage/current signal (E, $\mathbf{H}$ field), by rotating a reference point $\mathrm{P}$ (in which the EM field is defined) around the dielectric corner. In Figure 2 we report the equivalent position on the equivalent transmission line model, related to the EM field defined at a distance $\rho=\left|\mathbf{r}-\mathbf{r}^{\prime}\right|$, and by rotating of an angle equal to $\nu \phi$ around the dielectric corner. Figure 2(a) indicates three reference planes related to the EM field position: the first one (position Ref. [1]) is defined on the dielectric interface, the second
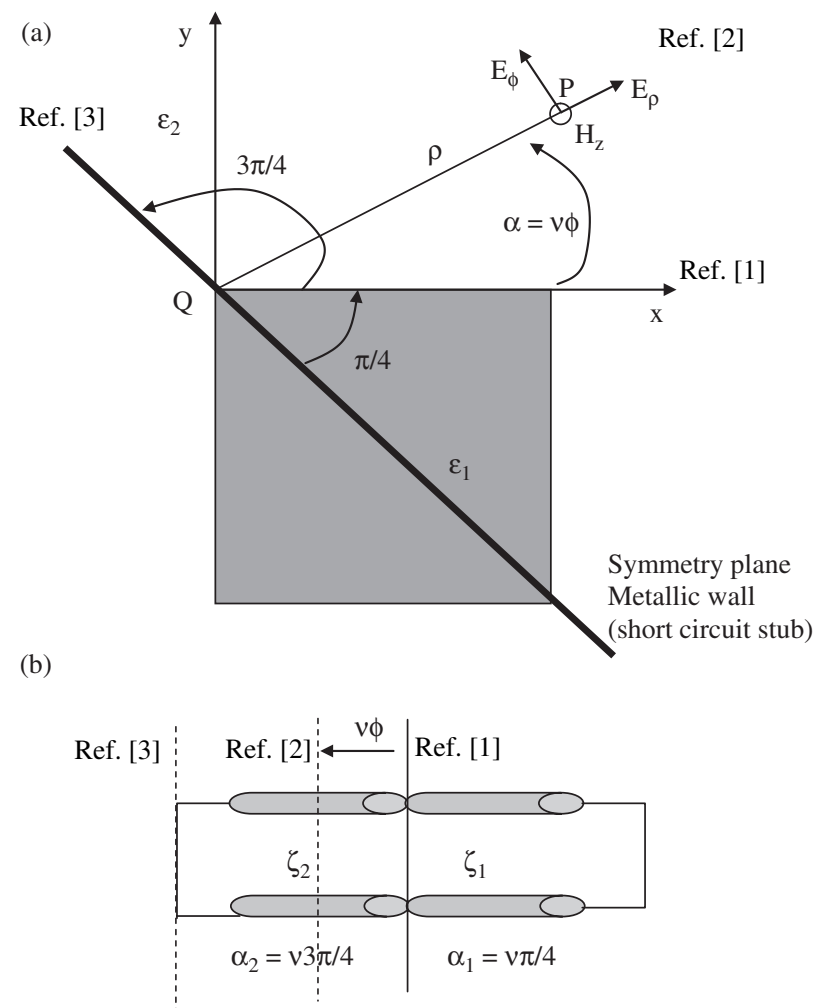

Fig. 2. TE $\mathrm{TE}^{z}$ field: (a) EM field components and symmetry plane (metallic wall); (b) EM field position on the equivalent transmission line. 
one on a generic position defined by $\rho$ (position Ref. [2]), and finally the last one (position Ref. [3]) on the metallic wall symmetry plane. Figure 2(b) shows the equivalent position of the three reference planes defined above on the equivalent transmission line model. We observe that Figure 2(b) represents the equivalent transmission line model with short circuit stub, obtained by the even property of the $H_{z}$ component related to a $\mathrm{TE}^{z}$ field. By making use of the continuity of the EM field for the TE case (continuity of the $H_{z}$ and $E_{\rho}$ component) one derives the following $\mathrm{ABCD}$ representation ${ }^{11}$

$$
\left(\begin{array}{c}
V_{i+1} \\
I_{i+1}
\end{array}\right)=\left(\begin{array}{cc}
\cos \left(\nu \phi_{i}\right) & \zeta_{i} \sin \left(\nu \phi_{i}\right) \\
\zeta_{i}^{-1} \sin \left(\nu \phi_{i}\right) & \cos \left(\nu \phi_{i}\right)
\end{array}\right)\left(\begin{array}{c}
V_{i} \\
I_{i}
\end{array}\right)
$$

where $\zeta_{i}=1 / \varepsilon$ is the normalized $\mathrm{TM}^{\phi}$ characteristic impedance. By introducing the load impedance $Z_{i}=V_{i} / I_{i}$ and $T_{i}=\tan \left(\nu \Psi_{i}\right)$, and by using the standard formula for the impedance transformation along a transmission line

$$
Z_{i}=\zeta_{i} \frac{Z_{i-1}+j \zeta_{i} T_{i}}{\zeta_{i}+j Z_{i-1} T_{i}} \quad i=1,2
$$

we can evaluate the resonance condition, ${ }^{11,12}$ and therefore the singularity $\nu$ of the electromagnetic field, by computing the total impedance of the transmission line circuit of Figure 2(b). In this case the transverse resonance condition, which provides the $\nu$ value, can be expressed by the following transcendental equation

$$
\frac{T_{1}}{\varepsilon_{1}}+\frac{T_{2}}{\varepsilon_{2}}=0
$$

By applying Eq. (7) to the configuration of the equivalent circuit of Figure 2(b) we obtain

$$
\nu=\frac{2}{\pi} \cos ^{-1}\left[\frac{\varepsilon_{2}-\varepsilon_{1}}{2\left(\varepsilon_{2}+\varepsilon_{1}\right)}\right]
$$

It is essential to note that, for the cubic symmetry, the three transmission lines produce the same resonance condition of Eq. (7). In fact, all the lines are connected at the same reference section and so they are characterized by the same electrical lengths $\alpha_{i}$.

\section{NEAR FIELD: MULTIPOLE EXPANSION OF THE VECTOR GREEN'S FUNCTION}

The electric and magnetic fields at an external point $P$ of Figure 1 are expressed as ${ }^{9,10}$

$\mathbf{E}(P)$

$$
\begin{aligned}
= & \frac{1}{j \omega 4 \pi \varepsilon_{c}} \nabla_{P} \times \nabla_{P} \times\left(\int_{V} \mathbf{J}_{i} \frac{e^{-\sigma \rho}}{\rho} d V_{Q}+\int_{S} \mathbf{H} \times \hat{n} \frac{e^{-\sigma \rho}}{\rho} d S_{Q}\right) \\
& -\frac{1}{4 \pi} \nabla_{P} \times\left(\int_{V} \mathbf{M}_{i} \frac{e^{-\sigma \rho}}{\rho} d V_{Q}+\int_{S} \hat{n} \times \mathbf{E} \frac{e^{-\sigma \rho}}{\rho} d S_{Q}\right)-\frac{\mathbf{J}_{i}}{j \omega \varepsilon_{c}}
\end{aligned}
$$

$$
\begin{aligned}
& \mathbf{H}(P) \\
& =\frac{1}{4 \pi} \nabla_{P} \times\left(\int_{V} \mathbf{J}_{i} \frac{e^{-\sigma \rho}}{\rho} d V_{Q}+\int_{S} \mathbf{H} \times \hat{n} \frac{e^{-\sigma \rho}}{\rho} d S_{Q}\right)+\frac{1}{j \omega \mu 4 \pi} \nabla_{P} \\
& \quad \times \nabla_{P} \times\left(\int_{V} \mathbf{M}_{i} \frac{e^{-\sigma \rho}}{\rho} d V_{Q}+\int_{S} \hat{n} \times \mathbf{E} \frac{e^{-\sigma \rho}}{\rho} d S_{Q}\right)-\frac{\mathbf{M}_{i}}{j \omega \varepsilon_{c}}
\end{aligned}
$$

The electric field of Eq. (9) can be rewritten as

$$
\begin{aligned}
\mathbf{E}(P)= & \frac{1}{j \omega 4 \pi \varepsilon_{c}} \nabla_{P} \times \nabla_{P} \\
& \times\left(\int_{V} \mathbf{J}_{i} G\left(\mathbf{r}, \mathbf{r}^{\prime}\right) d V_{Q}+\int_{S}(\mathbf{H} \times \hat{n}) G\left(\mathbf{r}, \mathbf{r}^{\prime}\right) d S_{Q}\right) \\
& -\frac{1}{4 \pi} \nabla_{P} \times\left(\int_{S}(\hat{n} \times \mathbf{E}) G\left(\mathbf{r}, \mathbf{r}^{\prime}\right) d S_{Q}\right)-\frac{\mathbf{J}_{i}}{j \omega \varepsilon_{c}} \\
= & \frac{1}{j \omega 4 \pi \varepsilon_{c}} \nabla_{P} \times \nabla_{P} \times\left(G(\mathbf{r}) \mathbf{P}_{0}+\int_{S} \mathbf{J}_{\mathbf{s}} G\left(\mathbf{r}, \mathbf{r}^{\prime}\right) d S_{Q}\right) \\
& -\frac{1}{4 \pi} \nabla_{P} \times\left(\int_{S} \mathbf{M}_{\mathbf{S}}\left(G\left(\mathbf{r}, \mathbf{r}^{\prime}\right)\right) d S_{Q}\right)-\frac{\mathbf{J}_{i}}{j \omega \varepsilon_{c}}
\end{aligned}
$$

The equivalence theorem ${ }^{9,11}$ provides on each facet the equivalent density currents $\mathbf{J}_{s i}=\mathbf{H} \times \mathbf{n}_{i}$, and $\mathbf{M}_{s i}=\mathbf{n}_{i} \times \mathbf{E}$ reported in Eq. (11) and in Figure 1(a). These density currents take into account the singularity factor $\nu$ evaluated through the STRD transmission line modeling. In the calculus of Eq. (11) we consider a multipole expansion of the Green $G\left(\mathbf{r}, \mathbf{r}^{\prime}\right)$ function in spherical harmonics is ${ }^{9,10-16}$

$$
G\left(\mathbf{r}, \mathbf{r}^{\prime}\right)=\sum_{l, m} g_{l}\left(r, r^{\prime}\right) Y_{l, m}^{*}\left(\theta^{\prime}, \varphi^{\prime}\right) Y_{l, m}(\theta, \varphi)
$$

where the functions $Y_{l, m}$ are defined as

$$
Y_{l, m}(\theta, \varphi)=\left[\frac{2 l+1}{4 \pi} \cdot \frac{(l-m) !}{(l+m) !}\right]^{1 / 2} P_{l}^{m}(\cos \theta) e^{j m \varphi}
$$

and $P_{l}^{m}$ are the Legendre functions ${ }^{9,17-18}$ given by

$$
\begin{array}{r}
P_{l}^{(m)}(x)=\frac{(-1)^{m}}{2^{l} l !}\left(1-x^{2}\right)^{m / 2} \frac{d^{l+m}}{d x^{l+m}}\left(x^{2}-1\right)^{l} \\
m=-l, \ldots,+l
\end{array}
$$

We observe that the expansion of Eq. (12) satisfies the orthogonality condition:

$$
\begin{aligned}
& \sum_{0}^{\infty}{ }_{l} \sum_{-l}^{+l} Y_{l, m}^{*}\left(\theta^{\prime}, \varphi^{\prime}\right) Y_{l, m}(\theta, \varphi) \\
& \quad=\delta\left(\cos \theta-\cos \theta^{\prime}\right) \delta\left(\varphi-\varphi^{\prime}\right)
\end{aligned}
$$

Index $l$ represents the order of the expansion and, in the case of three planar sources, its acceptable minimum value is $l=3$ (corresponding to three electric dipoles). Moreover the terms $g_{l}\left(r, r^{\prime}\right)$ of Eq. (12), given by

$$
g_{l}\left(r, r^{\prime}\right)=\left\{\begin{array}{ll}
-i k j_{l}(k r) h_{l}^{(2)}\left(k r^{\prime}\right) & r<r^{\prime} \\
-i k j_{l}\left(k r^{\prime}\right) h_{l}^{(2)}(k r) & r>r^{\prime}
\end{array} \quad(i=-j)\right.
$$


present a discontinuity peak of the first derivative at the point $Q$ (point of singularity reported in Fig. 1(a)). Moreover the Eq. (16) satisfies the Sommerfeld condition $\left(r g_{l} \rightarrow 0\right.$ for $\left.r \rightarrow \infty\right)$. The spherical Bessel functions ${ }^{9,19}$ in Eq. (16) are given by

$$
j_{l}(x)=\left(\frac{\pi}{2 x}\right)^{1 / 2} J_{l+(1 / 2)}(x)
$$

and the spherical Hankel functions ${ }^{9,20}$ are defined by

$$
h_{l}^{(2)}(x)=\left(\frac{\pi}{2 x}\right)^{1 / 2}\left[J_{l+(1 / 2)}(x)-i N_{l-(1 / 2)}(x)\right]
$$

that for a distance $r \ll \lambda$ (Refs. [7,8]) are of the type

$$
h_{l}^{2}(x) \simeq i \frac{1 \cdot 3 \cdot 5 \cdots(2 l-1)}{x^{l+1}}
$$

Regarding the contribution of the impressed currents $\mathbf{J}_{i}$ reported in Figure 1(a) we consider the three dipole moments generated by a cubic current wave source ${ }^{13}$ according to the $3 \mathrm{D}$ dielectric corner geometry. The three dipole moments in each direction are

$$
\mathbf{P}_{i 0}=\int \mathbf{J}_{i} d V=\mathbf{n}_{i} J_{i} L^{3} \frac{\sin (k L / 2)}{(k L / 2)}
$$

where $\mathbf{n}_{i}$ is the unit vector normal to the coordinate planes and $L$ is the side of the dielectric cube. The dipole moments values are obtained by applying the following impressed currents in the dielectric cube centre.

$$
\begin{gathered}
\mathbf{J}_{i_{x}}(\mathbf{r})=\mathbf{n}_{3} J_{i} e^{-j k x} \quad \mathbf{J}_{i_{y}}(\mathbf{r})=\mathbf{n}_{2} J_{i} e^{-j k y} \\
\mathbf{J}_{i_{z}}(\mathbf{r})=\mathbf{n}_{1} J_{i} e^{-j k z}
\end{gathered}
$$

\section{ANALYTICAL AND NUMERICAL RESULTS}

By applying the STRD for a $90 \mathrm{deg}$. dielectric corner embedded in air $\left(\varepsilon_{2}=1\right)$ we obtain the analytical

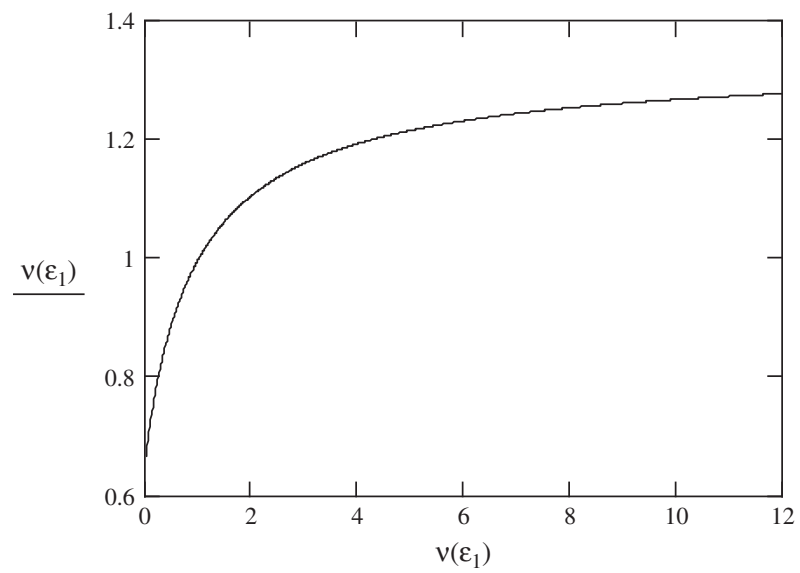

Fig. 3. Analytical behaviour of the singularity $\nu$ respect to the index $\varepsilon_{1}$. The index $\varepsilon_{2}$ is equal to 1 (air dielectric permittivity).

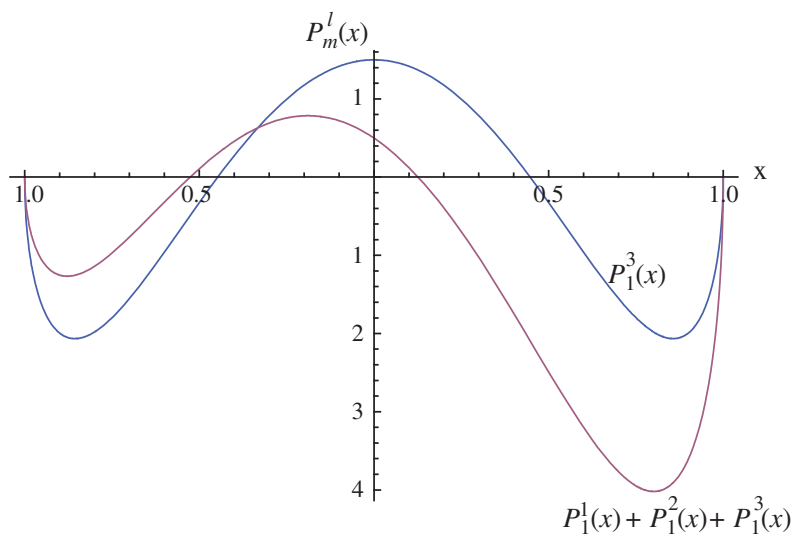

Fig. 4. Legendre functions.

behaviour of the singularity $\nu$ (see Fig. 3). In order to evaluate the electric field in a point $P$ near a $S_{i}\left(\varepsilon_{1}=10.49\right)$ dielectric cube corner we consider the Green $G\left(\mathbf{r}, \mathbf{r}^{\prime}\right)$ function expanded by the Legendre functions $P_{l}^{m}$. In Figure 4 we show the analytical Legendre functions used in the Green's expansion. The expanded Green's function in air material at working wavelength of $\lambda_{0}=1.55 \mu \mathrm{m}$ is reported in Figures 5 and 6. Figure 7 shows the electric near field $|E(P)|$ along the Si cube diagonal by using the
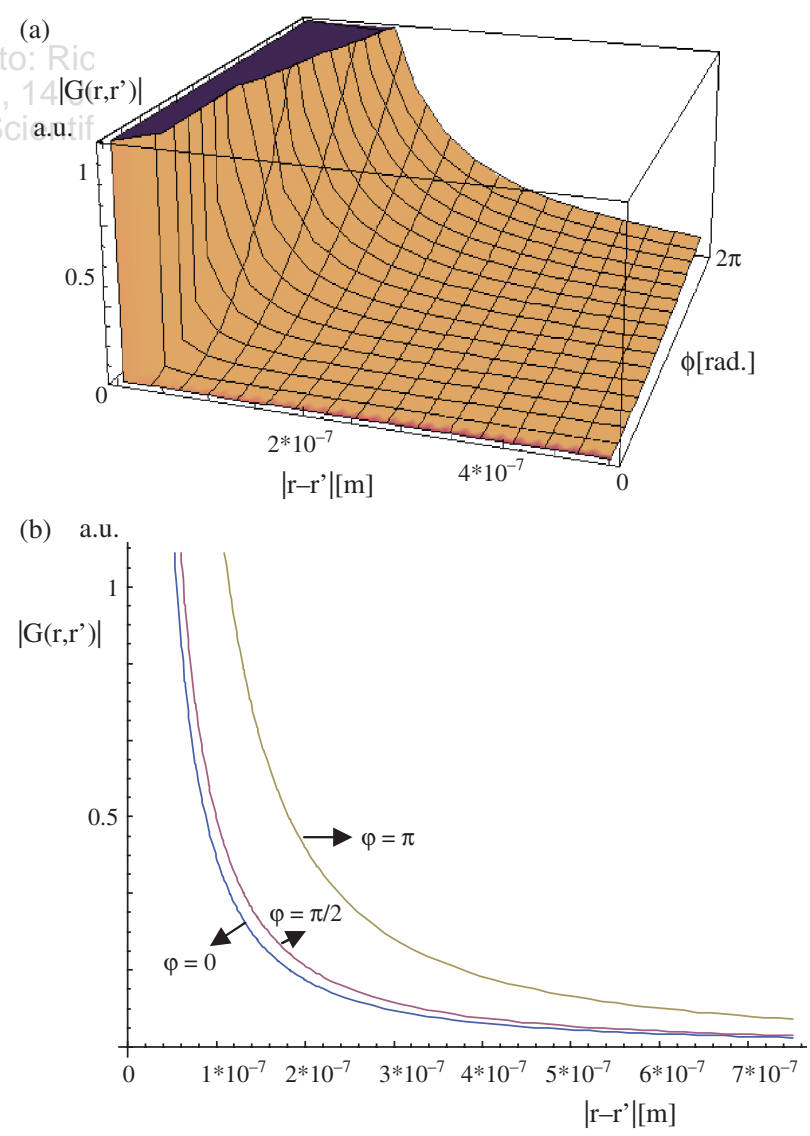

Fig. 5. (a) Analytical Green's function by varying the cylindrical coordinates $\varphi$ and $\rho=\left|\mathbf{r}-\mathbf{r}^{\prime}\right|$, (b) analytical Green's function for different values of $\varphi$ angles. 


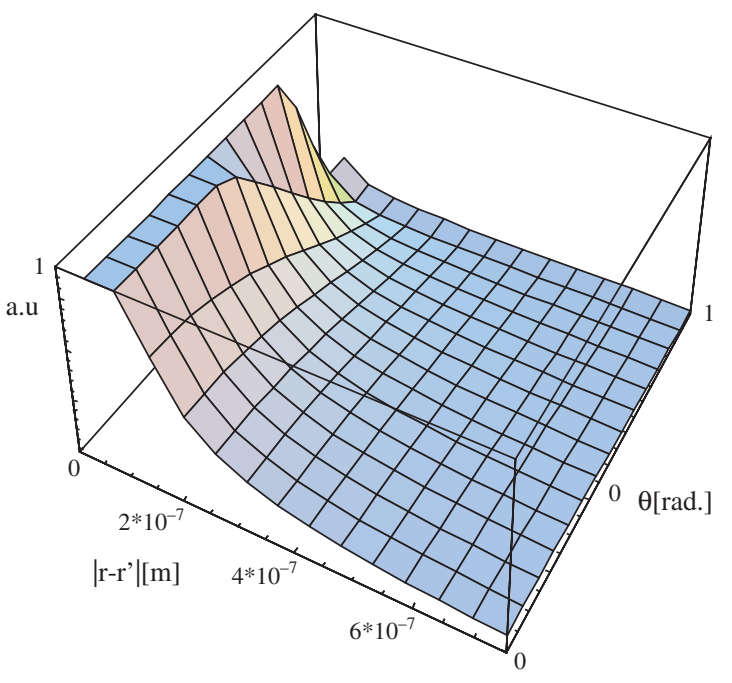

Fig. 6. Analytical Green's function by varying the cylindrical coordinates $\theta$ and $\rho=\left|\mathbf{r}-\mathbf{r}^{\prime}\right|$.

multipole expansion and the singularity $\nu$ evaluated by the STRD approach: a Si cubic dielectric corner $(L=1 \mu \mathrm{m})$ at working wavelength of $\lambda_{0}=1.55 \mu \mathrm{m}$ is considered. In order to validate STRD approach, we model a 3D dielectric corner by the FEM method. The model takes into account the Si dielectric cube $(L=10 \mu \mathrm{m})$ embedded in an air cube (cubical spatial domain closed by absorbing plates), each side of the dielectric cube is excited by surface currents $\mathbf{J}_{s}$ and $\mathbf{M}_{s}$ obtained from the internal impressed currents $\mathbf{J}_{i}$ at $\lambda_{0}=1.55 \mu \mathrm{m}$ defined in the analytical model. Through this model we evaluate the near field in proximity of the dielectric corner along $\mathrm{Si}$ cube diagonal. In order to obtain a good numerical resolution we use in the commercial FEM solver a large number of tetrahedral elements (total number of 612455 elements). In order to compare the results we consider a spatial

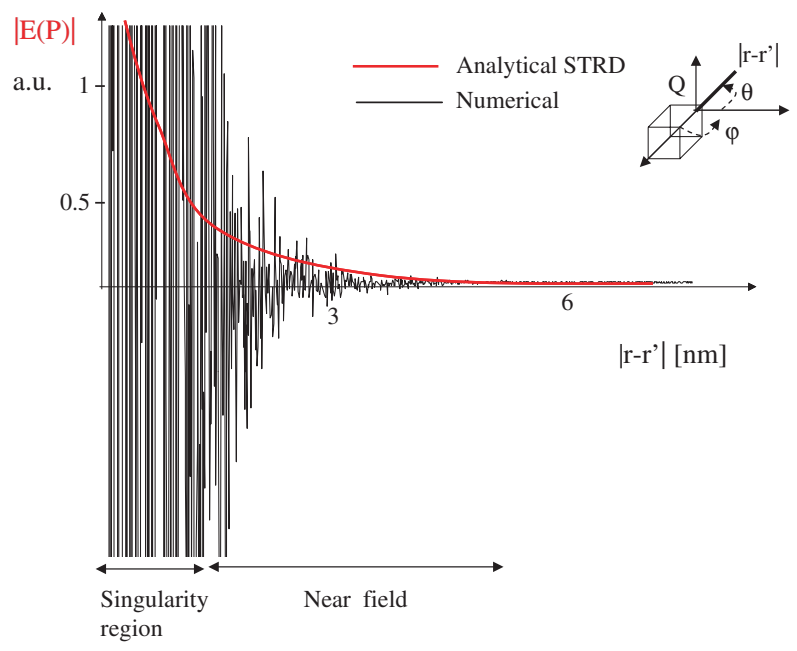

Fig. 7. Analytical and numerical electric near field $|E(P)|$ : the electric field is evaluated along the diagonal of the Si dielectric cube. Inset: cylindrical coordinates.

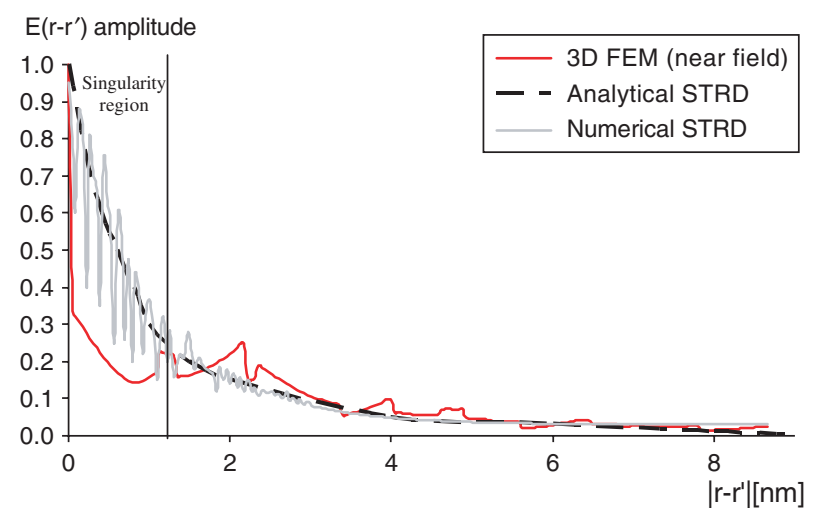

Fig. 8. Comparison between the near field development $E\left(r-r^{\prime}\right)$ the 3D FEM (commercial solver) model and the STRD model along the cube diagonal.

discretisation of the STRD method of the same length of tetrahedral elements used in the 3D FEM simulation. We observe from Figure 8 the comparison between the near field obtained by the 3D FEM modeling and by the STRD model: in both cases after few nanometers (singularity region) the field becomes stable by confirming the accuracy of the STRD model. Moreover the numerical STRD results oscillates around the analytical solution with a low numerical error. The efficiency of the STRD model is proved by the low computational cost of the numerical STRD approach: the multipole expansion order $(l=3)$ provides a good convergent solution by allowing to decrease the computational time of 12 times respect to the traditional FEM numerical modeling (1 hour is the computational time for the STRD model, and 12 hours is the computational time of the commercial FEM solver). We compared the central processing unit time (CPU) for a $1-\mathrm{GHz}$ 512/M-RAM personal computer.

\section{CONCLUSION}

We present a simple and accurate analysis of the 3D-dielectric cube corner obtained by the joint use of a multipole expansion and the Simultaneous Transverse Resonance Diffraction (STRD) approach. The STRD method can be also applied to a generic dielectric discontinuity, by providing a good characterization of the field scattered around a discontinuous region. The theory is validated by means of a numerical FEM simulation. The proposed model is suitable for inclusion in a standard full-wave numerical technique, (e.g., FDTD and FEM), in which a typical drawback is the accurate modeling of singularities that occur close to sharp edges.

\section{References}

1. L. Cascio, G. Tardioli, T. Rozzi, and W. J. R. Hoefer, IEEE Trans. on Microw. Theory and Tech. 44, 2519 (1996).

2. L. Cascio, G. Tardioli, T. Rozzi, and W. J. R. Hoefer, Microwave Symposium Digest, IEEE MTT-S International Symposium 1, 317 (1997). 
3. J. B. Keller, R. M. Lewis, and B. D. Seckler, J. Appl. Phys. 28,570 (1957).

4. H. S. Tan and D. K. Cheng, J. Math. Physics 10, 1957 (1969).

5. J. B. Keller, R. M. Lewis, and B. D. Seckler, J. Appl. Phys. 28,570 (1957).

6. E. A. Kraut and G. W. Lehman, J. Math. Phys. 10, 1340 (1969).

7. P. M. Morse and H. Feshbach, Methods of Theoretical Physics, edited by McGraw-Hill, New York (1953), pp. 1273-1283.

8. G. R. Hadley, IEEE J. Lightwave Technol. 20, 1221 (2002).

9. C. G. Someda, Onde Elettromagnetiche, UTET, Torino (1996).

10. V. I. Okhmatovski and A. C. Cangellaris, IEEE Trans. Antennas Propagat. 51, 3209 (2003).

11. T. Rozzi and M. Mongiardo, Open Electromagnetic Waveguides, IEE Electromagnetic Waves Series 43, London (1997), pp. 21-50.
12. L. Pierantoni, A. Massaro, and T. Rozzi, IEEE Trans. Microw. Theory Tech. 53, 1856 (2005).

13. I. V. Lindell, IEEE Trans. Antennas Propagat. 35, 683 (1987).

14. W. W. Lui, C.-L. Xu, W.-P. Huang, K. Yokoyama, and S. Seki, IEEE J. Lightwave Technol. 17, 1509 (1999).

15. A. Hausner, IEEE Trans. on Vis. and Comput. Graph. 3, 12 (1997).

16. J. Song and W. C. Chew, IEEE Microw. Wirel. Comp. Lett. 11, 311 (2001).

17. Y. Chen, T. L. Simpson, and T. Q. Ho, IEE Proceedings-H 139, 7 (1992).

18. J. S. Gardner, IEEE Trans. Ant. Propagat. 55, 1797 (2007).

19. M. S. Narasimhan and S. Christopher, IEEE Trans. Ant. Propagat. 32, 1268 (1984).

20. R. Cicchetti and A. Faraone, IEEE Trans. Ant. Propagat. 55, 1887 (2007).

Received: 20 October 2008. Accepted: 21 November 2008. 